inquiry into the exceptional character of the latter half of the year 1912. During that period the intensity of solar radiation appeared generally to be considerably below the values previously found; the decrease was indeed so marked that it could be detected in the records from the Campbell-Stokes instrument which is designed primarily for the registration of duration of sunshine. The diminution has been attributed to the presence in the atmosphere of an exceptional amount of fine dust arising from the volcanic eruption of Katmai, in Alaska, at the end of June, I9I2.

An ingenious explanation of the way in which the dust may stop the solar radiation without keeping in the earth's radiation to anything like the same degree has been put forward by Humphreys, in the Bulletin of the Mount Weather Observatory. The particles of dust have a diameter almost certainly greater than the wave-lengths of the most intense solar radiation, and smaller than the wave-lengths of terrestrial or atmospheric radiation, so that they would reflect the former but merely scatter the latter; and Humphrey's calculations show that the reflection would be much more effective than the scattering. Thus the effect on the temperature at the earth's surface is the reverse of that due to an increase in the absorbing power of the atmosphere, such as would be produced by increasing the water vapour or carbon dioxide in it. The theory is both novel and important; it indicates a method by which purely terrestrial agencies may profoundly affect the mean temperature of the globe, which may be sufficient justification for this digression.

The measurements at Warsaw bear out those found at other places; the intensity of radiation was slightly above the average for the earlier months of I9I2, but from July to the end of the vear it was nearly 20 per cent. below the average. The amount of the deficiency decreased in the first three months of I9r3, and thenceforward the radiation appears to have been about normal. The latest values given are those for July, I9I3.

The only other year in the period during which the records show a deficiency comparable with that for 1912 was 1903 (and the last two months of 1902), after the eruptions of Mont Pelée, Santa Maria, and Colima. It will be remembered that 1903 was a year remarkable in this country for its excessive rainfall and its disturbance of meteorological statistics and theories of periodicity.

The results of the whole series of measurements are discussed very fully for different altitudes of the sun, different times of day, and different conditions of the atmosphere, especially as regards humidity. The main text is in Polish, but the headings of the tables are given also in French, and there is a summary in French at the end of the paper.

\section{E. Gold}

\section{AMERICAN RESEARCH ON CLAYS. ${ }^{1}$}

$A$ CONSIDERABLE amount of interesting work $\mathrm{A}$ in connection with clays and the clay industries
has been done in recent years in Germany and in America, and no one has worked more enthusiastically than Messrs. Ashley and Bleininger. In Germany, too, Drs. Rieke and Endell are doing really fine work. The untimely death of the writer of the first-named pamphlet-Mr. H. E. Ashley-was a sad loss which must have considerably retarded subsequent developments. The clay industries the world over owe the Bureau of Standards, etc., in the United States a debt

1 H. E. Ashley, Technical Control of the Colloidal Matter of Clays; G. H. Brown, The Function of Time in the Vitrification of Clays; A. V' Bleininger and E. T. Montgomery. Effect of Overfiring upon the Structure of Clays. Three Technological Papers of the Bureau of Standards. (Washington, D.C., U.S.A, rgr3.)

NO. 2327 , VOL. 93] of gratitude for having set aside such men as Messrs. Ashley and Bleininger to devote their whole time to this work, and the results must be a source of satisfaction to the authorities responsible for the innovation.

The posthumous pamphlet by Mr. Ashley, together with his "The Colloidal Matter of Clay and its Measurement" (I909), form a kind of monograph, or rather a brief advocating the colloidal theory as an explanation of the many curious properties of clays. Mr. Ashley was an extremist, and in consequence we $r$ c ve here probably the best possible statement of the theory without those doubts and difficulties which perplex and hamper less enthusiastic temperaments. For that reason, Mr. Ashley's brief is particularly valuable, even though it is certain that some of the applications of the colloidal theory will not be able to stand, in their present form, before adverse criticism.

The colloidal theory has been mainly directed to explaining the plasticity of clays. The argument appears to run somewhat as follows:-The plasticity of clays is determined by the contained colloidal matter (and also by the degree of fineness of the constituent particles of the clay). The greater the plasticity, the greater the proportion of colloids. Colloids are always present in clays in unknown quantities, and the proportion of colloids in clays of different plasticity varies in accord with theoretical requirements! The amount of colloidal matter in a clay is assumed to be proportional to the dye-absorptive power of the clay, and this, in turn, is stated to be proportional to the plasticity.

As a matter of fact, the real plasticity of a clay is not so easily measured. The potter's thumb is the ultimate test, and any process of measurement must express by number those complex sensations which the potter "feels" when he estimates the plasticity of a clay. If measurements of the dye-absorptive power and of the fineness of the grain of a clay will do this, then the problem is solved in a most simple and interesting manner. Unfortunately, the method breaks down completely in practice. Consequently, we cannot really go further than this: the known facts favour the colloidal theory as the best qualitative explanation of plasticity yet suggested, but no one has succeeded in satisfactorily demonstrating the theory quantitatively. Thus we return to the view held by a writer in the eighteenth century, who stated that "the plasticity is due to the presence of a greasy medium between the particles of the clay." It is difficult to see how the plasticity of clays can be measured unless it be treated as a mechanical problem; and to the present writer, Zschokke's analysis of plasticity is far and away the greatest advance that has yet been made.

The two other pamphlets seem to be an application to American clays of some ideas suggested by the present writer in several papers a few years ago: "On the Speed of Vitrification of Clays," etc. Clays are made up of a heterogeneous mixture of particles of different sizes and composition; when clay productsbricks, etc. - are being fired, the more refractory particles start dissolving in the matrix formed by those which melt first. In the extreme case, the whole would form a homogeneous vitreous mass. The firing is stopped before this condition is reached. The stage at which the process of vitrification is arrested is determined by the nature of the required products-porcelain, firebricks, etc.-and on the character of the particular clay "body" being used. Each clay has its own specific character, and this explains how a fire. man with no sound principles to guide him-but a triumph of empiricism with one, or maybe two, types of clay-often fails ignominously when he is transferred from one district to another, using a different type of clay. 